\title{
ApoE4 genotype and cognitive performance in Korean health examinees with subjective memory complaints: A retrospective cross-sectional study
}

\author{
Eun-Hee $\mathrm{Nah}^{1 *}$, Seon $\mathrm{Cho}^{1}$, Suyoung $\mathrm{Kim}^{1}$, Jieun $\mathrm{Chu}^{1}$ and Han-Ik Cho ${ }^{2}$ \\ ${ }^{1}$ Health Promotion Research Institute, Korea Association of Health Promotion, Seoul, Korea \\ ${ }^{2}$ Medi Check Lab, Korea Association of Health Promotion, Cheongju, Korea
}

\begin{abstract}
Introduction: Subjective memory complaints are a natural phenomenon in the aging process. The apolipoprotein E epsilon 4 (ApoE4) allele is the strongest genetic risk factor for Alzheimer's disease. The aims of this study were to determine the distribution of the ApoE genotype and how the status of ApoE4 is associated with the cognitive function of Korean health examinees with subjective memory complaints.

Methods: A retrospective cross-sectional study was conducted with 1,107 health examinees with subjective memory complaints who underwent both medical and cognitive testing including ApoE genotyping at two health-promotion centers in two Korean cities between 2016 and 2017 . ApoE was genotyped using the Seeplex ApoE ACE Genotyping (Seegene, Seoul, Korea). Cognitive function was assessed using the K-MMSE. All of the subjects were classified according to the ApoE4 status into non-carriers, heterozygotes, and homozygotes. The odds ratios (ORs) were estimated using logistic regression for assessing the relationship between the ApoE4 genotype and cognitive impairment. ANOVA was used to compare the cognitive domain scores according to the ApoE4 status.
\end{abstract}

Results: ApoE4 allele frequency was $10.6 \%$. ApoE4 homozygosity was significantly associated with a lower K-MMSE score ( $\leq 23)(\mathrm{OR}=4.651,95 \% \mathrm{CI}=1.287-$ 16.812) compared to ApoE4 non-carriers, and this association remained after adjusting for age, sex, BMI, blood pressure, blood lipid, and glucose. The scores in the temporal orientation and recall domains were lower in ApoE4 heterozygotes than in ApoE4 non-carriers $(P<0.05)$.

Discussion: ApoE4 homozygosity was associated with cognitive impairment in health examinees with subjective memory complaints and ApoE4 allele selectively affects to temporal orentation and recall domains.

\section{Introduction}

Alzheimer's disease $(\mathrm{AD})$ is a neurodegenerative brain disorder and the main cause of dementia. The duration of disease progression can range from several years to decades [1]. There is a transitional period between normal aging and the clinical diagnosis of AD. Although subjective memory complaints are a natural phenomenon in the aging process, it may be in this transitional zone such as subjective cognitive decline (SCD). SCD is a possible predictor of future cognitive decline [2]. Although individuals with SCD would remain stable or revert to a normal state [3], undetermined genetic factors are partly responsible for SCD progressing to dementia [4].

Genetically, the apolipoprotein E epsilon 4 (ApoE4) allele is the strongest genetic risk factor for $\mathrm{AD}$. The ApoE gene has three common alleles (E2, E3, and E4). ApoE4 carriers are at higher risks of both earlyonset $\mathrm{AD}$ and late-onset $\mathrm{AD}$ compared with ApoE3 carriers $[5,6]$. With regard to nondemented people, there is controversy about the association between risk factors for cognitive impairment and ApoE4. Some studies [7-9] have observed ApoE4-related deficits in cognitive performance, while control studies $[10,11]$ have found no difference in the prevalence of ApoE4 between healthy controls and SCD groups.

In addition, ApoE4 genotype distribution in not only the general population but also $\mathrm{AD}$ varies between different geographical regions [12]. For example, Norberg et al. [13] showed that the ApoE4 prevalence exhibits a north-south gradient across European both healthy people and $\mathrm{AD}$, being highest in the north and lowest in the south.

The aims of this study were to determine the distribution of the ApoE genotype and how the status of ApoE4 is associated with the cognitive function of Korean health examinees with subjective memory complaints. Moreover, the cognitive domain scores were evaluated according to ApoE4 status.

\section{Methods}

\section{Subjects}

A retrospective cross-sectional study was conducted with 1,107 health examinees with subjective memory complaints who underwent both medical and cognitive testing including ApoE genotyping as part of a health checkup at two health-promotion centers in two Korean cities between January 2016 and December 2017. The exclusion criteria

*Correspondence to: Eun-Hee Nah, Health Promotion Research Institute, Korea Association of Health Promotion, 396, Gonghang-daero, Gangseo-Gu, Seoul 07649, Korea, E-mail: cellonah@hanmail.net

Key words: apolipoprotein E, apoE4 genotype, cognition, cognitive domain, subjective memory complaints

Received: May 13, 2019; Accepted: May 27, 2019; Published: May 30, 2019 
for this study were being previously diagnosed as $\mathrm{AD}$ or the presence of brain tumors, head trauma, or stroke. The study subjects gave their informed consent for cognitive testing and ApoE genotying.

\section{Cognitive assessments}

All subjects were assessed for cognitive function using the Korean version of Mini-Mental State Examination (K-MMSE) [14]. The MMSE is a widely used screening test for assessing cognitive abilities, and it was modified and translated into Korean to suit the cultural background of Koreans. The K-MMSE includes items assessing orientation (5 points for temporal orientation and 5 points for spatial orientation), registration ( 3 points), recall ( 3 points), attention and calculation (5 points), and language and praxis ( 2 points for naming, 3 points for oral command comprehension, and 1 point each for repetition, reading, writing, and visuospatial ability). The maximum K-MMSE score is 30, and higher scores indicate higher cognitive function. A K-MMSE score of $\leq 23$ was defined as cognitive impairment [15].

\section{ApoE genotyping}

Whole peripheral blood was collected in EDTA tubes. Genomic DNA was extracted from the peripheral blood using SEEPREP 12 (DiaSorin, Dublin, Ireland) according to the manufacturers' protocol. ApoE was genotyped as recommended in the manufacturer's instructions using the Seeplex ApoE ACE Genotyping (Seegene, Seoul, Korea). Amplification has performed using TC-96/G/H(b)/A (Hangzhou Bioer Technology, Hangzhou, China) and the SEEAMP PCR System (Seegene, Seoul, Korea). Briefly, $3 \mu \mathrm{L}$ of DNA extract in $17 \mu \mathrm{L}$ of multiplex PCR Mastermix was amplified. ApoE genotypes were detected using the LabChip DX System (Caliper Life Sciences, Hopkinton, USA). The analysis yielded the following patterns: $158 \mathrm{Cys}$ and 112Cys for E2/E2; 158Cys, 112Cys and 158Arg for E2/E3; 158Cys, 112Arg, 112Cys and 158Arg for E2/E4; 112Cys and 158Arg for E3/E3; 112Arg, 112Cys and 158Arg for E3/E4; and 112Arg and 158Arg for E4/ E4.

\section{Statistical analysis}

The study subjects were classified into ApoE4 non-carriers (E2/E2, $\mathrm{E} 3 / \mathrm{E} 3$, and E2/E3), ApoE4 heterozygotes (E2/E4 and E3/E4), and ApoE4 homozygotes (E4/E4). Data are presented as mean \pm standard deviation or percentage for categorical variables. Analysis of variance (ANOVA) with Scheffe's multiple-comparison tests and the chi-square test were used to compare the characteristics according to the ApoE4 status. Univariate and multivariate (adjusted) logistic regression analyses were performed to evaluate the association between ApoE4 status and cognitive impairment. The multivariate models were implemented with adjustment for age, sex, body mass index (BMI), blood pressure, blood lipid levels, and fasting blood glucose level. ANOVA or Welch's ANOVA (in case of heterogeneity of the variance assumption) was used to compare the cognitive domain scores according to the ApoE4 status. Statistical analyses were performed using SAS version 9.4 (SAS Institute, Cary, NC, USA). A $P$ value of $<0.05$ was considered statistically significant.

\section{Results}

These 1,107 examinees comprised 301 males and 806 females aged $66.2 \pm 8.3$ years (range $38-89$ years). The most frequent allele was ApoE3 (82.5\%), followed by ApoE4 (10.6\%) and ApoE2 (6.9\%). The ApoE3/ E3 genotype was the most common (68.4\%), followed by ApoE3/E4 (17.2\%), ApoE2/E3 (11.1\%), ApoE4/E4 (1.4\%), ApoE2/E4 (1.3\%), and ApoE2/E2 (0.7\%).

\section{Characteristics of study subjects according to ApoE4 status}

The overall K-MMSE score was 26.2 \pm 3.1 and was lower in ApoE4 homozygotes than in ApoE4 non-carriers $(P=0.002)$. Cognitive impairment was more common in ApoE4 homozygotes than in ApoE4 non-carriers and ApoE4 heterozygotes $(P=0.018)$. Blood triglyceride levels were higher in ApoE4 heterozygotes and ApoE4 homozygotes than in ApoE4 non-carriers $(P=0.022)$ (Table 1$)$.

\section{Association of ApoE4 status with cognitive impairment}

While age, sex, and systolic blood pressure were associated with cognitive impairment in a univariate model, this association of systolic blood pressure disappeared in multivariate models. ApoE4 homozygotes were significantly associated with cognitive impairment, and this association remained after adjusting for age, sex, BMI, blood pressure, blood lipid levels and fasting blood glucose level (odds ratio

Table 1. Characteristics of study subjects by ApoE genotype

\begin{tabular}{|c|c|c|c|c|c|c|c|c|c|}
\hline \multirow[b]{3}{*}{ K-MMSE } & \multirow{2}{*}{\multicolumn{2}{|c|}{$\begin{array}{c}\text { ApoE4 Negative } \\
\qquad(\mathrm{N}=\mathbf{8 8 8})\end{array}$}} & \multirow{2}{*}{\multicolumn{2}{|c|}{$\begin{array}{c}\text { ApoE4 } \\
\text { Heterozygote }\end{array}$}} & \multirow{2}{*}{\multicolumn{2}{|c|}{$\begin{array}{c}\text { ApoE4 } \\
\text { Homozygote }\end{array}$}} & \multirow{2}{*}{\multicolumn{2}{|c|}{$\begin{array}{c}\text { Total } \\
(\mathrm{N}=\mathbf{1 , 1 0 7 )}\end{array}$}} & \multirow{3}{*}{$\begin{array}{r}P \text { value } \\
0.002\end{array}$} \\
\hline & & & & & & & & & \\
\hline & 26.3 & $\pm 3^{b}$ & 25.7 & \pm 3.4 & 24.1 & $\pm 4.2^{\mathrm{a}}$ & 26.2 & \pm 3.1 & \\
\hline $\begin{array}{c}\text { K-MMSE } \leq 23 \\
(\mathrm{~N}, \%)\end{array}$ & 126 & $(14.2)$ & 41 & (20.1) & 5 & $(33.3)$ & 172 & $(15.5)$ & 0.018 \\
\hline Age, yr & 66.2 & \pm 8.4 & 66.0 & \pm 7.8 & 68.7 & \pm 6.7 & 66.2 & \pm 8.3 & 0.472 \\
\hline Male (N, \%) & 245 & $(27.6)$ & 48 & $(23.5)$ & 8 & $(53.3)$ & 301 & $(27.2)$ & 0.036 \\
\hline BMI, $\mathrm{kg} / \mathrm{m}^{2}$ & 24.5 & \pm 3.2 & 24.5 & \pm 3.2 & 23.2 & \pm 2.1 & 24.5 & \pm 3.1 & 0.281 \\
\hline SBP, mmHg & 122.1 & \pm 13.8 & 122.1 & \pm 14.2 & 122.4 & \pm 18.6 & 122.1 & \pm 13.9 & 0.997 \\
\hline DBP, $\mathrm{mmHg}$ & 74.2 & \pm 8.4 & 73.5 & \pm 9 & 75.2 & \pm 8.2 & 74.1 & \pm 8.5 & 0.577 \\
\hline $\mathrm{TC}, \mathrm{mmol} / \mathrm{L}$ & 5.07 & \pm 0.99 & 5.09 & \pm 1.05 & 5.01 & \pm 0.83 & 5.07 & \pm 1.00 & 0.953 \\
\hline $\mathrm{TG}, \mathrm{mmol} / \mathrm{L}$ & 1.21 & \pm 0.73 & 1.39 & \pm 0.77 & 1.25 & \pm 0.48 & 1.25 & \pm 0.74 & 0.022 \\
\hline $\mathrm{HDL}, \mathrm{mmol} / \mathrm{L}$ & 1.50 & \pm 0.37 & 1.43 & \pm 0.36 & 1.38 & \pm 0.42 & 1.49 & \pm 0.37 & 0.057 \\
\hline $\mathrm{LDL}, \mathrm{mmol} / \mathrm{L}$ & 3.01 & \pm 0.89 & 3.04 & \pm 0.98 & 3.09 & \pm 0.78 & 3.01 & \pm 0.9 & 0.880 \\
\hline FBS, mmol/L & 5.68 & \pm 1.34 & 5.86 & \pm 1.34 & 5.58 & \pm 1.78 & 5.72 & \pm 1.35 & 0.278 \\
\hline $\begin{array}{l}\mathrm{HbA} 1 \mathrm{c}, \mathrm{mmol} / \\
\mathrm{mol}\end{array}$ & 42.3 & \pm 12.0 & 42.6 & \pm 9.8 & 40.7 & \pm 9.0 & 42.3 & \pm 11.6 & 0.877 \\
\hline
\end{tabular}

Data are mean \pm standard deviation or $N(\%)$ values.

BMI, body mass index; SBP, systolic blood pressure; DBP, diastolic blood pressure; TC, total cholesterol; TG, total triglycerides; HDL, high-density lipoprotein cholesterol; LDL, lowdensity lipoprotein cholesterol; FBS, fasting blood glucose; HbAlc, hemoglobin A1c.

$P$ value derived from one-way ANOVA and chi-square test was used for intergroup comparison.

a, b,: Different letters indicate a significant intergroup difference based on Scheffe's multiple-comparison test. 
$[\mathrm{OR}]=4.651,95 \%$ confidence interval $[\mathrm{CI}]=1.287-16.812)$. In ApoE4 heterozygotes, this association disappeared after adjusting for age, sex, BMI, blood pressure, blood lipid levels and fasting blood glucose level (Table 2).

\section{Cognitive domain scores according to ApoE4 status}

Table 3 presents the cognitive domain scores according to ApoE4 status. ApoE4 heterozygotes had significantly lower scores for temporal orientation $(P=0.025)$ and recall domains $(P=0.017)$ compared to ApoE4 non-carriers. In ApoE4 homozygotes, those cognitive domains showed lower scores compared to ApoE4 non-carrier and heterozygotes, but the differences did not reach statistical significance (Table 3).

\section{Discussion}

This study found that ApoE4 homozygosity was associated with cognitive impairment in health examinees with subjective memory complaints, and that this association persisted after adjusting for age, sex, and cardiovascular risk factors. Several domains of cognitive performance differed according to the ApoE4 status. ApoE4 heterozygotes had lower scores in the temporal orientation and recall domains compared to ApoE4 non-carriers.

The frequency of ApoE4 was $10.6 \%$ in our study. The prevalence of ApoE4 genotype has varied in previous studies. The human ApoE2, ApoE3, and ApoE4 alleles have worldwide frequencies of $8.4 \%$, $77.9 \%$, and $13.7 \%$, respectively [16]. However, while the frequency of ApoE4 was $13.5 \%$ in an elderly Brazilian population [9], it was $8.7 \%$ in a community-dwelling Korean population [17]. Differences in the study subjects and methods used for ApoE genotyping may also have contributed to the variations in the reported prevalence rates of the ApoE4 genotype.
The effects of the ApoE genotype on the increased risk of $\mathrm{AD}$ are thought to be mediated by differential effects of ApoE4 on amyloid- $\beta$ deposition in the brain [18]. The general effects of ApoE4 that result in cognitive deficits also include ApoE4-related impairment to neuronal integrity and repair mechanisms as well as cardiovascular-related effects of the ApoE4 genotype [19]. ApoE4 carriers are at an increased risk of not only $\mathrm{AD}$ associated with an increased risk of cerebral amyloid angiopathy, but also age-related cognitive decline during normal aging [20]. Healthy ApoE4 carriers who are not diagnosed with mild cognitive impairment (MCI) or AD show accelerated longitudinal declines in memory tests, revealing the possibility of a pre-MCI state in ApoE4 carriers [21]. This association of ApoE4 with cognitive decline showed many years before cognitive impairment becomes clinically apparent in study using a mixed model for longitudinal change with age [22]. In cognitively normal people, ApoE4 is associated with enhanced amyloid pathology, which increases the amount of amyloid deposition, in a genedose-dependent manner [23]. A previous meta-analysis [24] found that cognitive impairment was associated with ApoE homozygosity but not heterozygosity. In our study, ApoE4 homozygosity was associated with cognitive impairment, and this association persisted after adjusting for age, sex and cardiovascular risk factors. However, this association with cognitive impairment in ApoE4 heterozygotes disappeared after adjusting for cardiovascular risk factors such as BMI, blood pressure, fasting blood glucose level and blood lipid levels. These findings suggest that there could be the gene-dose-dependent manner of relationship between ApoE4 genotype and its effect on cognitive impairment.

The effect of ApoE4 on the cognitive performance domains was selective in our study. Several studies [25,26] have observed decrements in episodic memory function among ApoE4 carriers, with a suggested underlying mechanism being ApoE4 carriers having a smaller hippocampal volume. Moreover, Yip et al. [27] reported that

Table 2. Association between ApoE4 status and cognitive impairment (K-MMSE score $\leq 23$ )

\begin{tabular}{|c|c|c|c|c|c|c|}
\hline & \multirow{2}{*}{\multicolumn{2}{|c|}{ Univariate model }} & \multicolumn{4}{|c|}{ Multiple model } \\
\hline & & & \multicolumn{2}{|c|}{ Model 1} & \multicolumn{2}{|c|}{ Model 2} \\
\hline & OR & $(95 \%$ CI $)$ & OR & $(95 \%$ CI $)$ & OR & $(95 \% \mathrm{CI})$ \\
\hline \multicolumn{7}{|l|}{ Allele E4(ref=0) } \\
\hline 1 & 1.521 & $(1.029,2.249)$ & 1.498 & $(0.938,2.394)$ & 1.507 & $(0.933,2.435)$ \\
\hline 2 & 3.024 & $(1.017,8.993)$ & 3.887 & $(1.143,13.221)$ & 4.651 & $(1.287,16.812)$ \\
\hline Age, yr & 1.14 & $(1.112,1.169)$ & 1.139 & $(1.107,1.172)$ & 1.14 & $(1.107,1.174)$ \\
\hline Sex(ref. male) & 1.494 & $(1.007,2.215)$ & 2.147 & $(1.326,3.479)$ & 2.205 & $(1.339,3.632)$ \\
\hline BMI, $\mathrm{kg} / \mathrm{m}^{2}$ & 0.983 & $(0.928,1.041)$ & 0.974 & $(0.913,1.039)$ & 0.961 & $(0.897,1.029)$ \\
\hline SBP, $\mathrm{mmHg}$ & 1.027 & $(1.014,1.04)$ & 1.013 & $(0.992,1.033)$ & 1.012 & $(0.991,1.033)$ \\
\hline DBP, $\mathrm{mmHg}$ & 1.019 & $(0.998,1.041)$ & 0.999 & $(0.967,1.033)$ & 1.002 & $(0.968,1.036)$ \\
\hline $\mathrm{TG}, \mathrm{mmol} / \mathrm{L}$ & 1.033 & $(0.817,1.307)$ & & & 0.93 & $(0.65,1.329)$ \\
\hline $\mathrm{HDL}, \mathrm{mmol} / \mathrm{L}$ & 0.676 & $(0.412,1.11)$ & & & 0.613 & $(0.319,1.178)$ \\
\hline $\mathrm{LDL}, \mathrm{mmol} / \mathrm{L}$ & 0.924 & $(0.757,1.129)$ & & & 1.036 & $(0.83,1.293)$ \\
\hline FBS, mmol/L & 1.077 & $(0.958,1.21)$ & & & 1 & $(0.855,1.169)$ \\
\hline
\end{tabular}

Data are odds ratio (OR) and $95 \%$ confidence interval $(\mathrm{CI})$ values.

Model 1: Adjusted for age, sex, and body mass index (BMI), and blood pressure.

Model 2: Adjusted for age, sex, BMI, blood pressure, blood lipid levels, and fasting blood glucose level.

BMI, body mass index; SBP, systolic blood pressure; DBP, diastolic blood pressure; TC, total cholesterol; TG, total triglycerides; HDL, high-density lipoprotein cholesterol; LDL, lowdensity lipoprotein cholesterol; FBS, fasting blood glucose.

Table 3. Cognitive domain scores according to ApoE4 status

\begin{tabular}{|c|c|c|c|c|c|c|c|}
\hline \multirow{2}{*}{\begin{tabular}{|c|} 
Domains \\
TemporL orientation*
\end{tabular}} & \multicolumn{2}{|c|}{ ApoE4 non-carriers $(\mathrm{N}=888)$} & \multicolumn{2}{|c|}{ ApoE4 heterozygotes $(\mathrm{N}=204)$} & \multicolumn{2}{|c|}{ ApoE4 homozygotes (N=15) } & \multirow{2}{*}{$\begin{array}{c}P \text { value } \\
0.025\end{array}$} \\
\hline & 4.70 & $\pm 0.71^{\mathrm{a}}$ & 4.5 & $\pm 1.02^{\mathrm{b}}$ & 4.4 & \pm 1.4 & \\
\hline Spatial orientation* & 4.56 & \pm 0.63 & 4.48 & \pm 0.74 & 4.2 & \pm 0.77 & 0.106 \\
\hline Registration & 2.96 & \pm 0.25 & 2.95 & \pm 0.24 & 3 & \pm 0 & 0.698 \\
\hline Attention and calculation & 3.67 & \pm 1.39 & 3.69 & \pm 1.39 & 3.07 & \pm 1.67 & 0.243 \\
\hline Recall* & 1.84 & $\pm 0.97^{\mathrm{a}}$ & 1.62 & $\pm 1.01^{\mathrm{b}}$ & 1.4 & \pm 1.3 & 0.017 \\
\hline Language and praxis* & 8.57 & \pm 0.75 & 8.48 & \pm 0.9 & 8 & \pm 1.2 & 0.102 \\
\hline
\end{tabular}

Data are mean \pm standard deviation. *Using Welch's ANOVA in case of heterogeneity of the variances assumption. ${ }^{\mathrm{a}, \mathrm{b}}$ Different letters indicate a significant difference 
other ability domains such as primary memory (short-term memory) and visuospatial function were less affected among ApoE4 carriers. These findings are consistent with our results, in which ApoE4 carriers exhibited lower scores in the temporal orientation and delayed recall domains compared to ApoE4 non-carriers, but with no significant decrease in short-term memory domains such as registration and other domains. Contrary to our expectations, although the temporal orientation and delayed recall domains showed the lowest scores in ApoE homozygotes, the differences did not reach statistical significance. This lack of significance could have been due to the relatively small number of ApoE4 homozygotes.

A prospective study of a cognitively normal cohort showed that the risk of dementia in ApoE4 carriers is negatively associated with higher education, higher level of leisure activities, and the absence of vascular risk factors [28]. Moreover, Head, et al. [29] demonstrated that physical exercise is strongly associated with reduced positivity for Pittsburgh compound B-which indicates the presence of fibrillar aggregates of amyloid- $\beta$-in cognitively normal ApoE4 carriers. Those studies indicate that higher education, actively participating in leisure activities and exercise, and maintaining vascular health could be beneficial in reducing the risk of $\mathrm{AD}$ and cognitive decline, particularly among ApoE4 carriers. Although the ApoE genotype is an unmodifiable factor of cognitive impairment, knowledge of which ApoE genotype a person is carrying could be helpful in prevention or delaying cognitive impairment by promoting health behaviors in a targeted manner.

This study has some limitations. First, the cross-sectional design meant that the study could not reveal the causal relationship between ApoE genotypes and cognitive function. Also, potential confounding factors for cognitive function such as education, and the presence of depression were not adjusted. However, age, sex, and cardiovascular risk factors including BMI, blood pressure, blood lipid levels, and fasting blood glucose level were adjusted when evaluating the association between ApoE4 status and cognitive impairment. Second, cognitive function was measured using single cognitive measure, the K-MMSE. However, the K-MMSE is the most commonly used screening test in primary care units, including health-promotion centers. Finally, the preponderance of female participants $(73 \%)$ is another limitation of our study.

\section{Conclusion}

In conclusion, the present study involving health examinees with subjective memory complaints found that ApoE4 homozygosity was associated with cognitive impairment, after adjusting for age, sex and cardiovascular risk factors. ApoE4 carriers had significantly lower scores for temporal orientation and recall domains compared to ApoE4 non-carriers. Our results suggest that ApoE4 carriers with subjective memory complaints should receive close monitoring for the development of $\mathrm{AD}$ and recommendations to exercise and maintain vascular health in order to prevent or delay cognitive impairment.

\section{Funding}

This research received no specific grant from any funding agency in the public or commercial sectors.

\section{Conflicts of interest}

No potential conflicts of interest relevant to this article were reported.

\section{Ethics statement}

This study was approved by the Institutional Review Board of the Korea Association of Health Promotion (approval no. 130750-201807HR-016).

\section{ORCID}

Eun-Hee Nah http://orcid.org/0000-0003-0637-4364.

\section{References}

1. Burns A, Iliffe S (2009) Alzheimer's disease. BMJ 338: b158.

2. Reisberg B, Prichep L, Mosconi L, John ER, Glodzik-Sobanska L, et al. (2008) The pre-mild cognitive impairment, subjective cognitive impairment stage of Alzheimer's disease. Alzheimers Dement 4: S98-108.

3. Wolfsgruber S, Kleineidam L, Wagner M, Mösch E, Bickel H, et al. (2016) Differential Risk of Incident Alzheimer's Disease Dementia in Stable Versus Unstable Patterns of Subjective Cognitive Decline. J Alzheimers Dis 54: 1135-1146.

4. Mitchell AJ, Beaumont H, Ferguson D, Yadegarfar M, Stubbs B (2014) Risk of dementia and mild cognitive impairment in older people with subjective memory complaints: meta-analysis. Acta Psychiatr Scand 130: 439-451.

5. Corder EH, Saunders AM, Strittmatter WJ, Schmechel DE, Gaskell PC, et al. (1993) Gene dose of apolipoprotein E type 4 allele and the risk of Alzheimer's disease in late onset families. Science 261: 921-923.

6. Liu CC, Liu CC, Kanekiyo T, Xu H, Bu G (2013) Apolipoprotein E and Alzheimer disease: risk, mechanisms and therapy. Nat Rev Neurol 9: 106-118.

7. Anstey K, Christensen H (2000) Education, activity, health, blood pressure and apolipoprotein $\mathrm{E}$ as predictors of cognitive change in old age: a review. Gerontology 46: $163-177$.

8. Lautenschlager NT, Flicker L, Vasikaran S, Leedman P, Almeida OP (2005) Subjective memory complaints with and without objective memory impairment: relationship with risk factors for dementia. Am J Geriatr Psychiatry 13: 731-734.

9. Quintino-Santos SR, Lima-Costa MF, Uchoa E, Firmo JO, Moriguchi EH, et al (2012) Homozygosity for the APOE E4 allele is solely associated with lower cognitive performance in Brazilian community-dwelling older adults: the Bambuí Study. Braz J Psychiatr 34: 440-445.

10. Verdile G, Laws SM, Henley D, Ames D, Bush AI, et al (2014) Associations between gonadotropins, testosterone and $\beta$ amyloid in men at risk of Alzheimer's disease. Mol Psychiatry 19: 69-75.

11. Caselli RJ, Chen K, Locke DE, Lee W, Roontiva A, et al. (2014) Subjective cognitive decline: self and informant comparisons. Alzheimers Dement 10: 93-98.

12. Crean S, Ward A, Mercaldi CJ, Collins JM, Cook MN, et al. (2011) Apolipoprotein E $\& 4$ prevalence in Alzheimer's disease patients varies across global populations: a systematic literature review and meta-analysis. Dement Geriatr Cogn Disord 31: 20-30.

13. Norberg J, Graff C, Almkvist O, Ewers M, Frisoni GB, et al. (2011) Regional differences in effects of APOE $\varepsilon 4$ on cognitive impairment in non-demented subjects. Dement Geriatr Cogn Disord 32: 135-142.

14. Han C, Jo SA, Jo I, Kim E, Park MH, et al. (2008) An adaptation of the Korean minimental state examination (K-MMSE) in elderly Koreans: demographic influence and population-based norms (the AGE study). Arch Gerontol Geriatr 47: 302-310.

15. Park JH, Kwon YC (1989) Standardization of Korean Version of the Mini-Mental State Examination(MMSE-K) for Use in the Elderly. Part II. Diagnostic Validity. J Korean Neuropsychiatr Assoc 28: 508-513.

16. Farrer LA, Cupples LA, Haines JL, Hyman B, Kukull WA, et al. (1997) Effects of age, sex, and ethnicity on the association between apolipoprotein E genotype and Alzheimer disease: A meta-analysis. JAMA 278: 1349-1356.

17. Shin MH, Kweon SS, Choi JS, Lee YH, Nam HS, et al. (2014) The effect of an APOE polymorphism on cognitive function depends on age. J Neurol 261: 66-72.

18. Kok E, Haikonen S, Luoto T, Huhtala H, Goebeler S, et al. (2009) Apolipoprotein E-dependent accumulation of Alzheimer disease-related lesions begins in middle age. Ann Neurol 65: 650-657.

19. Smith JD (2002) Apolipoproteins and aging: emerging mechanisms. Ageing Res Rev 1: 345-365. 
Nah E (2019) ApoE4 genotype and cognitive performance in Korean health examinees with subjective memory complaints: A retrospective cross-sectional study

20. Dik MG, Jonker C, Comijs HC, Bouter LM, Twisk JW, et al. (2001) Memory complaints and APOE-epsilon4 accelerate cognitive decline in cognitively normal elderly. Neurology 57: 2217-2222.

21. Caselli RJ, Reiman EM, Locke DE, Hutton ML, Hentz JG, et al. (2007) Cognitive domain decline in healthy apolipoprotein E epsilon4 homozygotes before the diagnosis of mild cognitive impairment. Arch Neurol 64: 1306-1311.

22. Caselli RJ, Dueck AC, Osborne D, Sabbagh MN, Connor DJ, et al. (2009) Longitudina modeling of age-related memory decline and the APOE epsilon 4 effect. $N$ Engl J Med 361: $255-263$

23. Reiman EM, Chen K, Liu X, Bandy D, Yu M, et al. (2009) Fibrillar amyloid-beta burden in cognitively normal people at 3 levels of genetic risk for Alzheimer's disease. Proc Natl Acad Sci USA 106: 6820-6825.

24. Small BJ, Rosnick CB, Fratiglioni L, Bäckman L (2004) Apolipoprotein E and cognitive performance: a meta-analysis. Psychol Aging 19: 592-600.
25. Bondi MW, Salmon DP, Monsch AU, Galasko D, Butters N, et al. (1995) Episodic memory changes are associated with the APOE-epsilon 4 allele in nondemented older adults. Neurology 45: 2203-2206.

26. Wilson RS, Bienias JL, Berry-Kravis E, Evans DA, Bennett DA (2002) The apolipoprotein E epsilon 2 allele and decline in episodic memory. $J$ Neurol Neurosurg Psychiatry 73: 672-677.

27. Yip AG, Brayne C, Easton D, Rubinsztein DC (2002) Apolipoprotein E4 is only a weak predictor of dementia and cognitive decline in the general population. $J$ Med Genet 39: 639-643.

28. Ferrari C, Xu WL, Wang HX, Winblad B, Sorbi S, et al. (2013) How can elderly apolipoprotein E $\varepsilon 4$ carriers remain free from dementia? Neurobiol Aging 34: 13-21.

29. Head D, Bugg JM, Goate AM, Fagan AM, Mintun MA, et al. (2012) Exercise Engagement as a Moderator of the Effects of APOE Genotype on Amyloid Deposition. Arch Neurol 69: 636-643.

Copyright: $@ 2019$ Nah E. This is an open-access article distributed under the terms of the Creative Commons Attribution License, which permits unrestricted use, distribution, and reproduction in any medium, provided the original author and source are credited. 